\title{
ATROPINE-INDUCED CONVULSIONS IN THE SEPTOHIPPOCAMPAL SYSTEM. I. EFFECTS OF CANNULA POSITION AND SEX
}

\author{
Josef Herink \\ Purkyně Military Medical Academy, Hradec Králové; (Rector: doc. MUDr. S. Býma, CSc.)
}

Summary: Effects of local administration of atropine into the medial septal nucleus (MSN) and dorsal septal nucleus (DSN) were tested in laboratory rats. Atropine administration led to the development of a spike/wave activity in the hippocampus as well as amygdala within 10 minutes. The frequency of spikes ranged in the absolute values from 20 to 25 spikes per minute in the case of atropine administration to MSN, while in the case of DSN it reached only about 16 spikes per minute. Spike/wave activity outlasted in the EEG record within 2 hours. The average incidence of spikes was somewhat lower in females than in males. A possible relation of the atropine-induced spike/wave activity to another kinds of limbic convulsions is discussed.

Key words: Atropine; Convulsions; Rat; Septohippocampal system

\section{Introduction}

The septum, particularly the septohippocampal system, represents an important part of the limbic system. Regarding the present knowledge, its involvement in emotional processes, memory operations and in the course of movement automatisms may be assumed (15). The septum also participates in the formation of some types of electroencephalographic (EEG) activity. A typical example represents the production of 4 to $12 \mathrm{~Hz}$ activity - the "theta" rhythm (5).The medial septal nucleus (MSN) and the dorsal septal nucleus (DSN) - it is called also the nucleus of the diagonal band of Broca- are known to be the principal sources of cholinergic input to the hippocampal formation $(10,16)$. We have previously demostrated the importance of the both structures mentioned for the action of anticholinergic drugs (8). We have also demonstrated, by means of the local lesion strategy, the existence of some heterogenity within MSN and DSN. DSN is oriented most likely toward the limbic lobe and neocortical structures, while MSN is connected particularly with the hippocampal and amygdala region. Confirmation of this conclusion was based on the different changes in AChE activity following lesions of MSN and DSN (7). We have also provided additional evidence in this context in pharmacological experiments (9).

The participation of the septohippocampal system in the formation of some types of convulsive activity is also well-established $(16,17)$. A low seizure threshold of some parts of the limbic system predeterminates their relationship to some kinds of epilepsy. In particular, complex partial seizures are closely associated with the limbic system
(11). The muscarinic cholinergic agonist pilocarpine is capable in the case of its local administration to induce in rats limbic seizures and status epilepticus followed by a wide-spread propagation of epileptic activity to the cortex $(2,18)$. On the contrary, a lack information is concerned with the effect of local administration of anticholinergic drugs into the limbic system. In order to determine a possible ability of anticholinergic drugs to induce some kind of convulsive activity, we tested the effect of local administration of atropine into MSN and DSN.

\section{Material and Method}

Experiments were performed in 40 male and 10 female albino rats of the Wistar strain. All rats were anaesthetized throughout the experiments with thiopental sodium (50 $\mathrm{mg} / \mathrm{kg}$, intraperitoneally), and mounted into a stereotaxic apparatus with the bregma $1.0 \mathrm{~mm}$ above the lambda. After incision and retraction of the skin, connective tissue and muscles, trephine openings approximately $1.0 \mathrm{~mm}$ in diameter were placed onto the exposed skull unilaterally, in the case of the hippocampus and amygdala to the left with respect to the sagittal suture. The stereotaxic coordinates for recording needle electrodes were as follows: the dorsal hippocampus - $3.5 \mathrm{~mm}$ behind the bregma, $3.0 \mathrm{~mm}$ laterally to the sagittal suture (L), and $3.75 \mathrm{~mm}$ below the surface of the skull $(\mathrm{V})$, the central amygdala $-1.5 \mathrm{~mm}$ behind the bregma, $\mathrm{L}=2.5 \mathrm{~mm}, \mathrm{~V}=8.0 \mathrm{~mm}$. The stereotaxic coordinates for intracerebral cannula were as follows: MSN $0.75 \mathrm{~mm}$ before the bregma, $\mathrm{L}=0, \mathrm{~V}=5.5 \mathrm{~mm}$, DSN -0.75 $\mathrm{mm}$ before the bregma, $\mathrm{L}=0, \mathrm{~V}=4,25 \mathrm{~mm}$. Atropine was 
given by means of the intracerebral cannula in a dose of 0.2 $\mathrm{mg}$ in a volume of $1 \mu \mathrm{l}$ of saline. EEG activity was recorded at least 120 minutes following atropine administration, in parts of the experiments up to 240 minutes. Male rats were divided into four groups of 10 animals, i.e., two control groups with the administration of saline only into MSN or, respective, DSN, and two experimental groups with the administration of atropine into MSN or DSN. In the case of female rats only the effect of atropine administration into MSN was investigated. At the end of the experiments, the animals were given a lethal dose of thiopental sodium, and the brains were carefully removed for histological evaluation. The average frequency and amplitude of abnormal EEG phenomena was evaluated. The results are illustrated in Figs 5 and 6 as the mean and SD $(n=10)$. Statistical comparison was calculated by means of t-test.

\section{Results}

The atropine administration into both MSN and DSN led to the development of a spike or spike and wave activity in the hippocampus as well as amygdala. The first appearance of these spikes began on the background of EEG activity with a prevailing high amplitude around the 10th minute (Fig. 1. below). Spike and wave activity was fully developed in the course of $30-60$ minutes (Fig. 2, above) and outlasted within 2 hours. Its disappearance was frequently followed by high EEG activity with an occasional occurence of intermittent spikes or sharp waves (Fig. 2, below). Fig. 3 shows typical examples of spike and wave activity in the both structures observed at the interval of 15 and $120 \mathrm{mi}-$ nutes following atropine administration. In the case of the amygdala the wave, which followed the spike, was lower in comparison with the same wave recorded in the hippocampus. The whole complex of spike and wave in the hippocampus had a rather biphasic character, while the initial spike of a high amplitude dominated in the case of the amygdala. The amplitude of spikes in the hippocampus fluctuated mostly from 500 to $600 \mu \mathrm{V}$, the same aplitude in the amygdala was higher, i.e., from 800 to $1000 \mu \mathrm{V}$. It was not possible to induce this spike and wave activity in the proper septum, i.e., in the structure where stimulation was carried out (Fig. 4). The average incidence of spikes induced by atropine administration into DSN was lower within the entire group in comparison with the same incidence following atropine administration into MSN (Fig. 5). There was a difference between males and females with respect to MSN, the average incidence of spikes being somewhat lower in females in comparison with males (Fig. 6). However, this difference was less prominent than in case of MSN and DSN in the same sex (compare Fig. 5 and Fig. 6).

\section{Discussion}

Although the convulsive effect of atropine was described as early as 1973 , no attention has been given to this fin-

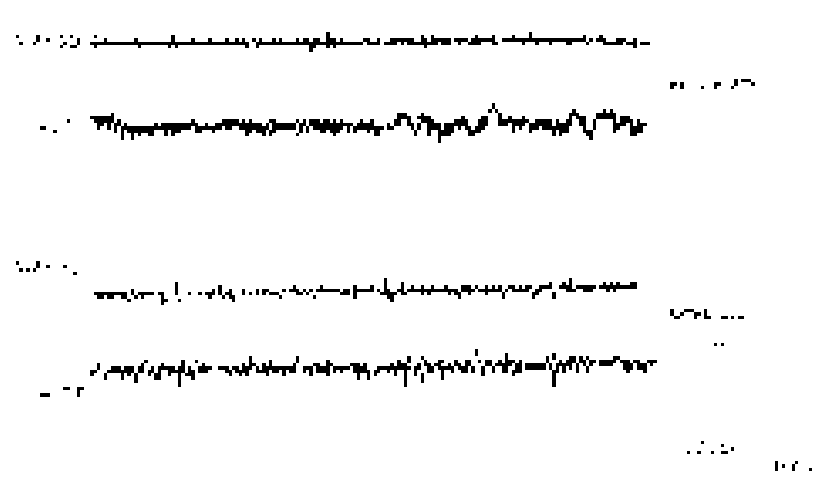

Fig. 1: EEG recordings from the left hippocampus (HIPP) and amygdala (AMYGD) before and after the administration of atropine (ATR) into MSN. Spiking begins about 10 minutes after atropine administration into both structures observed at the same time.

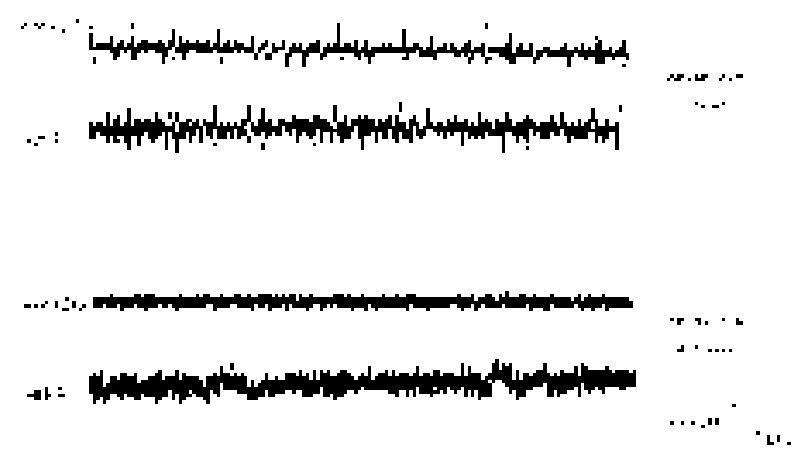

Fig. 2: EEG recordings from the left hippocampus and amygdala 60 and 240 minutes following atropine administration. Spike and wave activity is fully developed in the course of 30 - 60 minutes following atropine administration. Following disappearance of typical atropine-induced activity, sharp waves, or irregular spikes with periods of faster EEG background activity outlast in EEG recordings (Fig. 2, below).

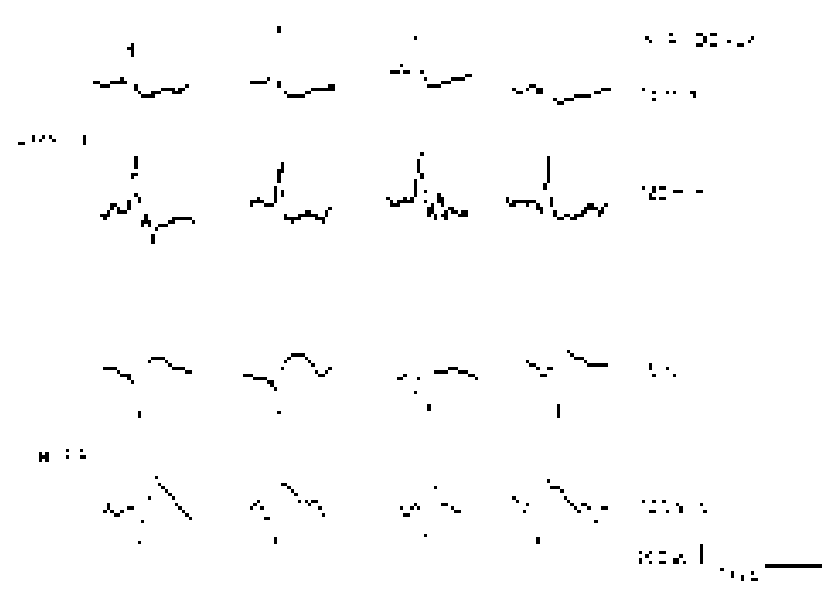

Fig. 3: Examples of various types of spike/wave activity in the amygdala (above) and hippocampus (below) 15 and 120 minutes following atropine administratione (in the text further details). 


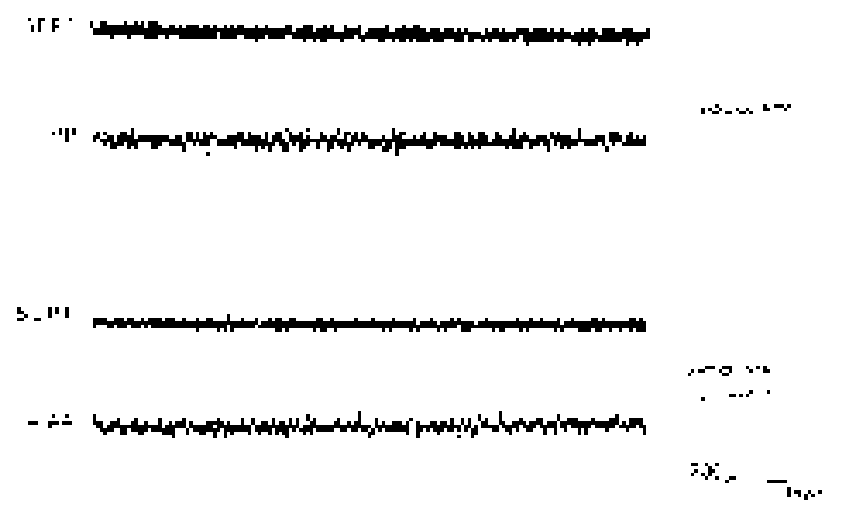

Fig. 4: EEG recordings from the hippocampus and medial septum (SEPT) before and after atropine administration into MSN. Note a lack of spiking in the proper septal area in comparison with the hippocampus.
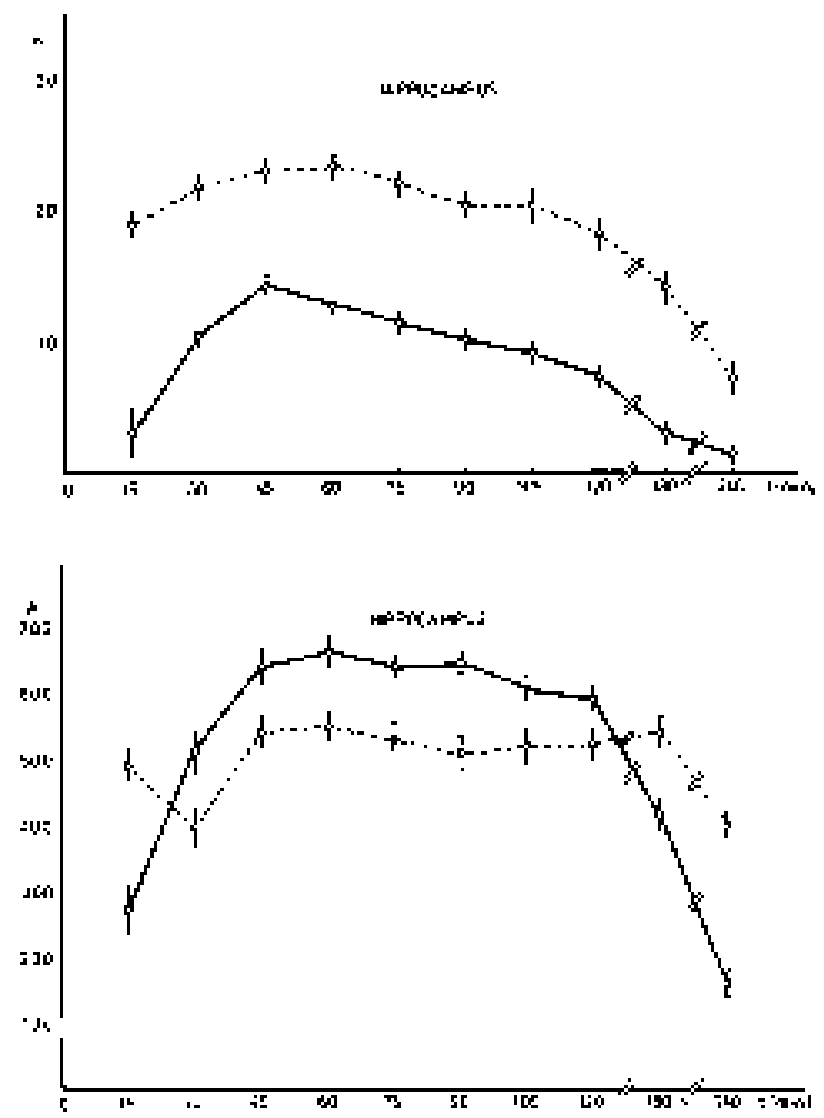

Fig. 5, above: Incidence of spike/wave activity in absolute values as the number of spikes per minute following administration of atropine into DSN (full line) and MSN (dashed line). Below: Amplitude of spike/wave activity as the mean and SD of $\mu \mathrm{V}$ following the administration of atropine into DSN (full line) and MSN (dashed line).
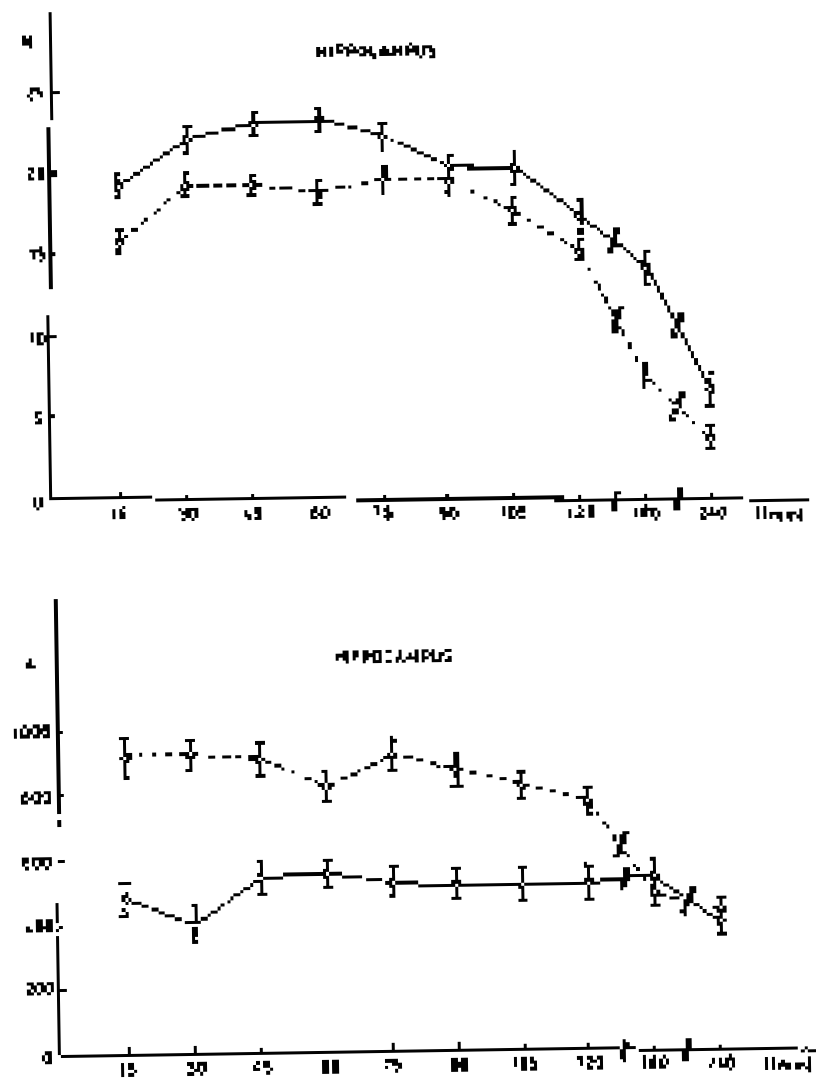

Fig. 6, above: Incidence of spike/wave activity in males (full line) and females (dashed line). Below: Amplitude of spike/wave activity in males (full line) and females (dashed line).

ding since. The exposition of the cat cortical surface to the effect of $1 \%$ solution of atropine led to the induction of prominent diphasic spikes, which occurred at a rate of approximately 1 per $0,5-3 \mathrm{~s}$. A few cats tested subsequently with a $2 \%$ solution of atropine developed more intense spike activity, which endured for hours (4). Although we used a different way of atropine administration into the different brain structures of different species, we surprisingly obtained a very similar pattern of spike and wave activity.

On the contrary,it is worth noticing that there exists a marked dissimilarity of the atropine-induced pattern in comparison with seizures produced in rodents by the administration of a cholinergic muscarinic agonist. Systemic or local administration of carbachol or pilocarpine results in bursts of spikes of much higher frequency than it is in the case of atropine administration (18). These bursts much more resemble electrically-induced afterdischarges of the „limbic“ type. The characteristic afterdischarge pattern consisted of a rapid „tonic“ phase, and a succesive „clonic“ phase with the occurrence of large, slow waves, occasionally with superimposed rapid activity $(6,12)$. This type of afterdischarges is considered as a model of generalized epileptic phenomena (14). On the contrary to this, a focal 
character of atropine-induced activity is quite noticeable. We can notice a certain degree of resemblance with spike and wave epizodes induced by low doses of pentylenetetrazol or gamma-hydroxybutyrate $(3,13)$. The natural endogenous GABA metabolite gamma-hydroxybutyrate is known to induce rhythmic spike and wave activity of a shape very similar to atropine-induced activity, as demonstrated in Fig. 3. However, there is a marked difference in the occurrence of both these types of spike and wave activity in the EEG patterns. While atropine-induced activity is quite regular in this sense and its frequency is as low as $0,33 \mathrm{~Hz}$, gammahydroxybutyrate-induced spikes show a cluster arrangement within EEG pattern and a higher rate of frequency.

Additional evidence is necessary to solve the problem of clasification of atropine-induced activity in relation to the other experimental models of epilepsy.

Acknowledgement:

Autor wish to thank Mrs. J. Bajgarová for skilfull technical assistance.

\section{References}

1. Bajgar J, Herink J, Hrdina V. Changes of acetylcholinesterase activity in the brain following septal lesions in the rat. Neuroscience, 1977;2:635-9.

2. Barone P, Parashos SA, Palma V, Marin C, Campanella $G$, Chase TN. Dopamine $D_{1}$ receptor modulation of pilocarpine-induced convulsions. Neuroscience 1990;34:209-17. 3. Brankačk J, Lahtinen H, Koivisto E, Riekkinen PJ. Epileptogenic spikes and seizures but not high voltage spindles are induced by local frontal cortical application of gamma-hydroxybutyrate. Epilepsy Res 1993;15:91-9.

4. Daniels JC, Spehlmann R. The convulsant effect of topically applied atropine. Electroencephal Clin Neurophysiol 1973;34:83-7.

5. Green JD. The hippocampus. Physiol Rev 1964;44:561608.

6. Handforth A, Ackermann RF. Hierarchy of seizure states in the electrogenic limbic status epilepticus model: Behavioral and electrographic observations of initial states and temporal progression. Epilepsia 1992;33:589-600.

7. Herink J, Bajgar J, Patočka J. Changes of acetylcholinesterase activity in some parts of the limbic system following septal lesions in rats. J Neurochem 1975;24:187-8.
8. Herink J, Fusek J, Hrdina V. Interaction of imipramine and physostigmine in the model of septal afterdischarges. Activ Nerv Sup (Praha) 1978;20:254-5.

9. Herink J, Koupilová M, Bajgar J. Protective effects of 7methoxytacrine in experimental models of epileptic activity. Homeostasis 1996;37:132-3.

10. Lewis PR, Shute CCD. The cholinergic limbic system: Projections to hippocampal formation, medial cortex, nuclei of the ascending cholinergic reticular system, and the subfornical organ and supraoptic crest. Brain 1967;90:521-40.

11. Lothman EW, Salerno RA, Perlin JB, Kaiser DL. Screening and characterization of antiepileptic drugs with rapidly recurring hippocampal seizures in rats. Epilepsy Res 1988;2:367-79.

12. Kapur J, Lothman EW. Loss of inhibition precedes delayed spontaneous seizures in the hippocampus after tetanic electrical stimulation. J Neurophysiol 1989;61: 427-34.

13. Kubová H, Mareš P. Effects of alprazolam on a model of human absences - rhythmic metrazol activity in rats. Physiol Res 1993;40:361-4.

14. Mareš P, Zouhar A. Do we possess adequate models of childhood epilepsies ? Physiol Bohemoslov 1988;37:1-9.

15. Soares JC, Mann JJ. The functional neuroanatomy of mood disorders. J Psychiatr Res 1997;31:393-432.

16. Stewart DJ, Vanderwolf CH. Hippocampal rhythmical slow activity following ibotenic acid lesions of the septal region. I. Relations to behavior and effect of atropine and urethane. Brain Res 1987;423:88-100.

17. Stewart DJ, Vanderwolf CH. Hippocampal rhythmical slow activity following ibotenic acid lesions of the septal region II. Changes in hippocampal activity during sleep. Brain Res 1987;423:101-8.

18. Turski L, Cavalheiro EA, Czuczwar SJ, Turski WA, Kleinrok Z. The seizures induced by pilocarpine:behavioral, electroencephalographic and neuropathological studies in rodents. Pol J Pharmacol Pharm 1987;39:545-5.

Submitted June 1998.

Accepted July 1998.

MUDr. Josef Herink, DrSc., Purkyně Military Medical Academy, 50001 Hradec Králové, Czech Republic. 